\title{
Space Charge Formation in Epoxy Resin Including Various Nanofillers
}

\author{
Alexis Hajiyiannis and George Chen \\ School of Electronics and Computer Science \\ University of Southampton, United Kingdom \\ Chao Zhang and Gary Stevens \\ GnoSys UK Ltd, University of Surrey, United Kingdom
}

\begin{abstract}
It has been widely anticipated that the combination of recent advances in nanocomposites technology with traditional and novel resin systems may create materials with enhanced electrical, thermal and mechanical properties. It has been recognised that charge dynamics under an electric field play an important role in determining the electrical performance of a material. In this research, the pulsed electroacoustic (PEA) technique has been used to measure space charge in epoxy anhydrite samples loaded with various nano-fillers. Space charge characteristics in both dried and wet samples have been measured at $\sim 27 \mathrm{kV} / \mathrm{mm}$. In addition to different charge dynamics, it has also been noticed that the electrical performance of nanocomposites has been affected in the presence of moisture. Further tests have been carried out at a lower field to reveal if different mechanisms take place.
\end{abstract}

\section{INTRODUCTION}

Nanodielectrics are very new in the electrical power industry and their properties still need to be studied and understood prior to their practical applications. A lot of research has been undertaken to reveal and understand the charge dynamics that govern the changed behaviours of these materials [1 - 19]. When these are understood, the materials can be ultimately optimized and therefore improve their dielectric performance as much as possible. The tiny size of the nanoparticles means that relative to their volume, their surface area is much greater compared with particles at the micron range such as in traditional epoxy resin insulation. This larger surface area will mean that whatever effects the interface between the fillers and the epoxy matrix have, they will be dominating the overall characteristics of the dielectric. This fact is widely accepted and a few models have been proposed recently. The models are all concerned with the effects produced by the interfaces between the nanoparticles and the composite's matrix. In the multi-core model $[8,19]$, the interface consists of 4 layers, i.e. (i) the bonded layer, (ii) the bound layer, (iii) the loose layer and (iv) an electric double layer which overlaps the first three layers. Lewis proposes an alternative model in [14] which aims to explain the behaviour of nanocomposites (polyethylene/titanium dioxide in particular) under the effect of electrical stress. The model assumes the nanoparticle to be a place where electron-hole recombination takes place and therefore where space charge is eliminated. The interface and the epoxy matrix around the nanoparticle serve as means of supplying transport paths for the negatively charged electrons and the positively charged holes to reach the nanoparticle and finally recombine.

Earlier research revealed that the inclusion of nanoparticles of $\mathrm{TiO} 2$ in LDPE can reduce the formation of space charge therefore leading to an improved electrical performance [10]. Similar observation was found in epoxy resin loaded with nanoparticles. It is speculated that the nanoparticle-matrix interface is considerably more conductive than the rest of the matrix unaffected by these interfaces [14]. These conductive areas, when partially overlapping, as in the case of nanodielectrics, can provide conductive paths for increased charge carrier mobility. This could result in more efficient charge dissipation and therefore increased dielectric breakdown strength [14]. A very important factor on the performance of epoxy dielectrics (and nanodielectrics) is the effect of moisture. When moisture is present, the properties of the dielectric change significantly, usually for the worse. A water shell model is proposed in $[4,11]$ to explain how the water behaves in the nanodielectric and the possible outcomes it can bring.

In the present paper space charge behaviours in both dried and wet epoxy resin filled with nano alumina and meso boron nitride have been investigated using the pulsed electroacoustic technique.

\section{EXPERIMENTAL DETAILS}

\section{A. Sample Preparation}

The epoxy resin system used in this study is a diglycidyl ether of bisphenol-A (DGEBA) epoxy resin cured with methyl hexahydro-phthalic anhydride and accelerated by tetraethylammonium bromide (TEAB). Nano aluminium oxide (nanoalumina) was obtained from Degussa with a particle size of about $13 \mathrm{~nm}$. This nanofiller has a nearly spherical shape with a specific surface area of about $100 \mathrm{~m}^{2} / \mathrm{g}$. The filler was dried extensively by thermal and vacuum treatment before use.

Epoxy nanocomposites were prepared by mixing the epoxy resin, anhydride hardener and filler by conventional mechanical stirring and degassed for $60 \mathrm{~min}$ at $60{ }^{\circ} \mathrm{C}$, the composites were then further mixed in an ultrasonic processor at $20 \mathrm{kHz}$ for typically 20 minutes. After that, the composites together with TEAB were mixed by stirring under degassing for $30 \mathrm{~min}$ again, and finally, they were cast in moulds for curing. Three types of epoxy nanocomposites were prepared 
with the concentration of alumina of 0,1 , and 5 wt $\%$, respectively. They were prepared as discs with a diameter of 35 $\mathrm{mm}$ and thickness of about $0.5 \mathrm{~mm}$. Detailed sample preparation can be found in [12] and the samples tested are listed in Table 1.

Table 1: Epoxy resin samples with nanofillers

\begin{tabular}{|l|l|}
\hline Specimens & Composition \\
\hline Unfilled epoxy & Epoxy/Anhydrite \\
\hline Epoxy -BN-5 & Epoxy/Anhydrite $+5 \mathrm{wt} \% \mathrm{BN}$ \\
\hline Epoxy-BN-10 & Epoxy/Anhydrite $+10 \mathrm{wt} \% \mathrm{BN}$ \\
\hline Epoxy-BN-20 & Epoxy/Anhydrite $+20 \mathrm{wt} \% \mathrm{BN}$ \\
\hline Epoxy-A-1 & Epoxy/Anhydrite $+1 \mathrm{wt} \%$ nanoalumina \\
\hline Epoxy-A-5 & Epoxy/Anhydrite $+5 \mathrm{wt} \%$ nanoalumina \\
\hline Epoxy-A-10 & Epoxy/Anhydrite $+10 \mathrm{wt} \%$ nanoalumina \\
\hline Epoxy-A-15 & Epoxy/Anhydrite $+15 \mathrm{wt} \%$ nanoalumina \\
\hline
\end{tabular}

BN denotes Boron Nitrite and A denotes Alumina

\section{B. The drying process}

It has been established that epoxy resin absorbs about $0.4 \%$ of its own weight in water when left at ambient conditions [13]. Once the samples are dried, their weight is monitored from the instance they are taken out of the oven so as to determine how fast they regain the lost water. The estimation $0.4 \%$ weight gain due to water absorption is confirmed only for the nanocomposites. It seems that the unfilled epoxy absorbs slightly higher than that $(\sim 0.54 \%)$. It is also observed that unfilled epoxy regains this water considerably faster than the nanocomposites.

Prior to the tests, the samples are placed in a vacuum oven at $130{ }^{\circ} \mathrm{C}$ for two hours to be dried. It has been shown in recent papers [4] that the addition of nanoparticles in epoxy dielectrics results in increased overall water absorption by the nanocomposite. It is not yet clear whether the "extra" water absorbed at the interfaces, due to the nanoparticles, can be extracted by any means.

\section{The PEA Technique}

The PEA technique is used to measure the space charge formation in the specimens under investigation. This technique has been accurately described in [20] and has been used extensively to measure the space charge present in several materials [21].

\section{EXPERIMENTAL RESULTS}

\section{A. Dried samples at $\sim 27 \mathrm{kV} / \mathrm{mm}$}

The tests on the above samples revealed the following observations:

- Although homo-charge is present in the unfilled resin, as the percentage loading increases, this homo-charge formed is considerably higher (see Figures 1 and 2).

- Local charge mobility seems to be greatly increased in the higher percentage nanocomposites. In epoxy_A_15, there was no charge present in the sample after 10 minutes that the DC field was switched off. In the unfilled resin, significant charge was present after 60 minutes.

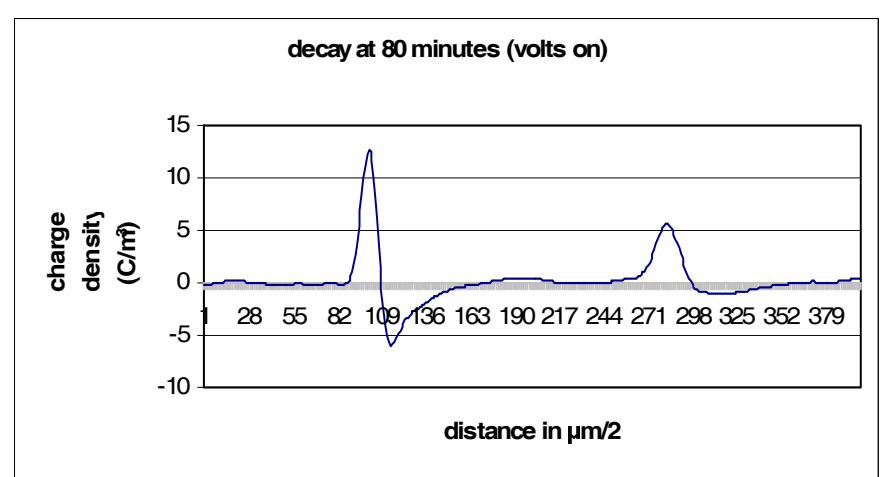

Fig.1 Residual charge present in unfilled epoxy at steady state and with the DC field off

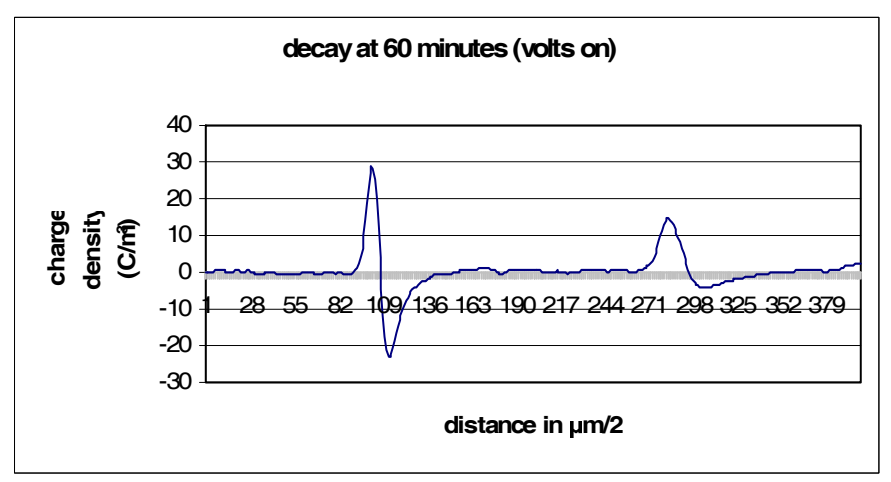

Fig.2 Residual charge present in epoxy_A_15 at steady state and with the DC field off

- $\quad$ Some charge forms in the bulk of the unfilled resin. This is not the case with the nanocomposites, especially with the higher percentage ones.

- Epoxy_BN_20 could not withstand the required DC field. This suggests that such a high loading of nanoparticles, cause the dielectric's characteristics to notably deteriorate. It was finally subjected to a lower field (25.5 $\mathrm{kV} / \mathrm{mm}$ ) in order to obtain some indicative results. Unexpectedly, the homo-charge observed was very low and nowhere near the one observed in epoxy_A_15.

\section{B. Undried samples at $\sim 27 \mathrm{kV} / \mathrm{mm}$}

The main characteristics observed with the undried samples are the following:

- Overall breakdown strength is severely reduced in the nanocomposites of lower than 5\% loading. Space charge readings could not be completely carried out due to breakdown of test sample.

- Charge mobility is increased in all the samples, but more notably in the lower percentage ones as shown in Fig. 3.

\section{Samples subjected to a field of $14 \mathrm{kV} / \mathrm{mm}$}

These tests were carried out to reveal any different mechanisms taking place when the nanodielectrics are subjected to a lower field than above. Generally, the trend observed is similar to the above. Higher homo-charge forms and increased charge mobility is observed with the higher 
percentage composites. A difference is that in most of the samples an overall charge formation is observed in the bulk, unlike in the nanodielectrics above. Another difference is that the charge decay rate (once the DC field is off) is considerably faster than the one observed when the samples were subjected to the higher field.

Total charge present in epoxy____10 at $11.2 \mathrm{kV}$

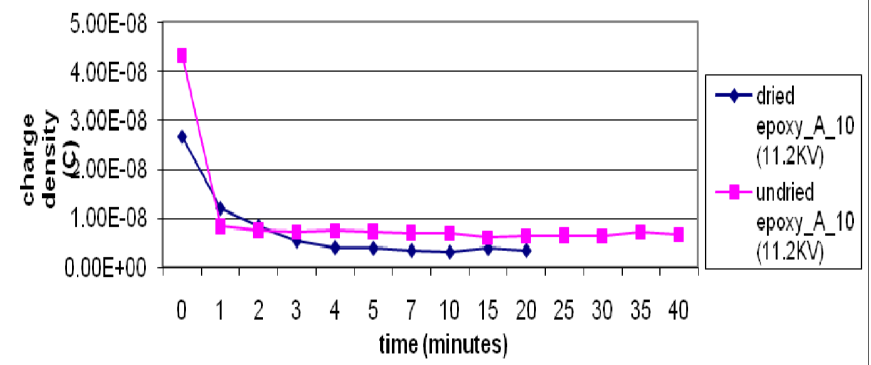

Fig.3 Charge decay of total charge present in dried and undried epoxy_A_10, showing the faster decay and the higher charge formed in the undried sample.

- Higher magnitude of charge is formed in the wet samples in majority of the cases compared with the dried ones as shown in Fig.4.

Total charge present al slendy siale - volts on (at. $27 \mathrm{kV} / \mathrm{nm}$ min)

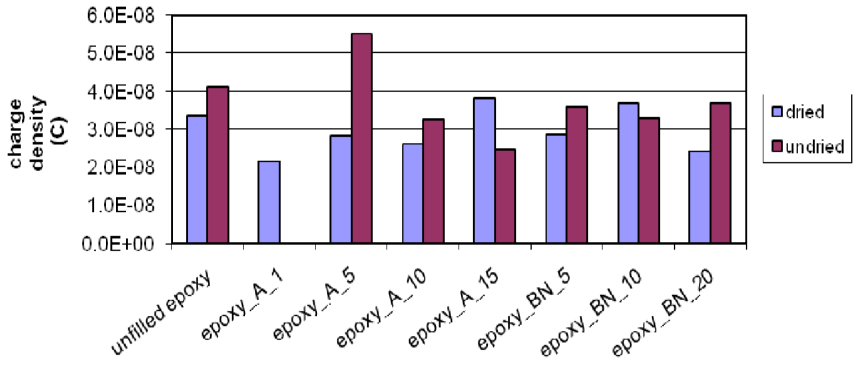

Fig.4 Total charge present in all the samples. In the majority of the readings, higher charge is formed in the wet samples

- The breakdown strength is not affected as much in the higher percentage samples. In fact, tests on epoxy_BN_20 were able to stand the whole testing period at $\sim 27 \mathrm{kV} / \mathrm{mm}$ without any interruptions.

\section{DISCUSSIONS}

\section{A. Increased charge mobility.}

In our previous paper [22], it was reported that the addition of nano alumina to LLDPE has three consequences. They are (i) enhancement of charge injection, (ii) ionisation possibly due to impurities of nanofillers and (iii) modification of charge trapping characteristics, leading to an increase in charge mobility. Charge distribution and dynamics become complicated as injection and ionisation show a different trend with the level of nanofillers and the applied voltage. Similar charge dynamics have been observed in the present study with epoxy resin nanocomposites. An increase in charge mobility with the level of nanofiller has also been observed.

Referring to Tanaka's multi-core model $[8,19]$, since the third layer is some 4 to10 times bigger than the first two layers, it will have the dominant effect on the overall bulk performance. It is assumed that this third layer is more conductive than the rest of the matrix and it is also speculated that aggregation of the nano and meso particles probably takes place in the higher percentage nanocomposites, resulting in different behaviour to that expected for isolated particles.

A simple calculation can be made. If we assume for nanoparticles concentrations around $20 \%$ b.w. (of $\sim 13 \mathrm{~nm}$ in diameter) and for a third layer thickness of $8 \mathrm{~nm}$ thick. The total affected volume would be around $46.5 \%$. It is therefore reasonable to assume that since such a high percentage of volume is being affected, it is highly possible that the shell layer interfaces overlap.

Even though the results reveal increased charge mobility, it should not be concluded that the overall conductivity of the nanodielectrics is increased. Besides, conductivity tests were not carried out. What can be deduced is that local charge mobility is increased and that charge accumulation is dissipated more quickly and effectively. Care must be taken so that the percolation limit is not exceeded because in that case, overall bulk conductivity would be increased. The increased local charge mobility can be very beneficial, since high stress areas within the dielectric are avoided $[1,3,9]$.

\section{B. Higher homo-charge formation}

Higher homocharge in nano-dielectrics was also observed in other papers $[1,4]$. Homo-charge is typically associated with charge injection. Under the influence of the applied field, the injected charges will tend to move towards the opposite electrode. Higher amount of homo-charge means that addition of nanofiller enhances charge injection. However, the higher amount of homo-charge could form a barrier to the injection of charge carriers in the sample. In the case of nanocomposites the higher mobility means that the injected charge can move quickly into the bulk region. On the other hand, the nanoparticles may act as recombining centres as suggested by Lewis [14]. This may explain the little charge presence in the centre of the nanocomposite sample compared with that of unfilled epoxy resin sample.

\section{Effect of water uptake}

Earlier, it was assumed that the epoxy resin absorbs $0.4 \%$ of its own weight in water when left at ambient conditions. It seems this might not be the case, and as found in [13], when present, nanoparticles cause higher water absorption. In our weighing results it was found that unfilled epoxy absorbed more water than the nanocomposites. It was also shown in [13] that samples that were wetted and dried again exhibited different characteristics than the virgin ones (not wetted at all). These facts draw the conclusion that once water is absorbed by the nanoparticle-matrix interface, it is difficult to be 
extracted again. Therefore, the interfaces are presumably constantly wet.

Charge mobility increases due to water being present. This phenomenon is more profound with the unfilled resin and the lower percentage composites. It is therefore re-confirmed that even when the matrix is wet, the interfaces (that are assumed to be constantly wet) are the predominant factors affecting the nanodielectric's behaviour.

It is worth noting that absorbed water can potentially have very different effects on nanocomposites that contain nanoparticles of different properties. If the nanoparticles are treated prior to manufacture, so as to be more hydrophobic, water absorption will probably be reduced. Also, the interface may now behave very differently and the overall dielectric characteristics will be altered.

\section{Differences in samples subjected to a field of $14 \mathrm{kV} / \mathrm{mm}$}

An overall negative charge is formed in most of the samples subjected to the lower field. Considering Lewis' model, it could be argued that the field was simply not high enough so that the electron-hole recombination at the nanoparticles could not be initiated. Therefore, the charge carriers in the bulk do not neutralise and the overall charge is observed.

It was noted that the charge decay in these samples was considerably faster than the samples subjected to the higher field. This could be the result of different kind of charge carriers causing the charge observed. In theory, free charge carriers, such as electrons and holes, should respond a lot more quickly than ions and dipoles in response to field changes. They are therefore likely to be the dominant charge carriers in these samples under charge injection conditions.

\section{CONCLUSION}

Space charge in epoxy resin filled with nano alumina and meso boron nitride has been investigated. The following conclusion may be drawn based on the experimental results and discussion presented.

Charge dynamics have been altered when nanoparticles are introduced into epoxy resin system. It seems that addition of alumina and boron nitrite enhances charge injection.

Charge profiles in the nanocomposites indicate that the nanoparticles may act as recombination centres, leading to little charge measured in the middle of sample bulk.

Moisture has a significant effect on charge dynamics and the overall performance of the nanocomposites.

\section{REFERENCES}

[1] J. C. Fothergill, J. K. Nelson, M. Fu "Dielectric Properties of Epoxy Nanocomposites containing $\mathrm{TiO}_{2}, \mathrm{AlO}_{3}$, and $\mathrm{ZnO}$ fillers" CEIDP, Boulder, Colorado, USA, pp. 406-409, 2004.
[2] N. Tagami, M. Okada, N. Hirai, T. Tanaka, Y. Ohki, T. Imai, M. Harada, M. Ochi "Effects of Curing and Filler Dispersion Methods on Dielectric Properties of Epoxy Nanocomposites" CEIDP, Vancouver, BC, Canada pp. 232-235, 2007.

[3] T. Tanaka, G. C Montanari, R. Mulhaupt "Polymer Nanocomposites as Dielectrics and Electrical Insulation-Perspectives for Processing Technologies, Material Characterization and Future Applications" IEEE Trans. on Diel. and Elec. Ins. Vol. 11, pp. 763-784, 2004.

[4] C. Zou, J. C. Fothergill and S. W. Rowe "The Effect of Water Absorption on the Dielectric Properties of Epoxy Nanocomposites" IEEE Tran.s on Diel. and Elec. Ins. Vol. 15, pg.106-116, 2008.

[5] R. C. Smith, C. Liang, M. Landry, J. K. Nelson and L. S. Schadler "The Mechanisms Leading to the Useful Electrical Properties of Polymer Nanodielectrics" IEEE Tran. on Diel. and Elec. Ins., Vol. 15, pp.187-196, 2008 .

[6] A. Cavallini, D. Fabiani, G.C Montanari "Time-evolution of Nanostructured Epoxy Resin due to Surface Partial Discharge Activities" CEIDP, Vancouver, BC, Canada, pp. 256-259, 2007.

[7] Y. Cao, P. C. Irwin and K. Younsi "The Future of Nanodielectrics in the Electrical Power Industry" IEEE Tran. on Diel. and Elec. Ins. Vol. 11, pp. 797-807, 2004.

[8] T. Tanaka, M. Kozako, N. Fuse and Y. Ohki, "Proposal of a multi-core model for polymer nanocomposite dielectric", IEEE TDEI, Vol.14, pp. 669 $681,2005$.

[9] T. Tanaka "Dielectric Nanocomposites with Insulating Properties" IEEE Tran. on Diel. and Elec. Ins. Vol. 12, pp. 914-928, 2005.

[10] M. S. Khalil, G. C. Montanari, D. Fabianl and F. Palmieri, "Investigation of the effect of titanium dioxide additive on space charge threshold field in polyethylene", $13^{\text {th }}$ Int. Symp. On High Voltage Eng. pp. 364, 2003.

[11] C. Zou, M. Fu, J.C Fothergill and S.W. Rowe "Influence of Absorbed Water on the Dielectric Properties and Glass-transition Temperature of Silicafilled Epoxy Nanocomposites" CEIDP pp. 321-324, 2006.

[12] C. Zhang, R. Mason and G. Stevens "Preparation, Characterization and Dielectric Properties of Epoxy and Polyethylene Nanocomposites" IEEJ Trans. FM, Vol. 126, pp. 1105-1111, 2006.

[13] C. Zhang and G. Stevens, "The Dielectric Response of Polar and Nonpolar Nanodielectrics”, IEEE TDEI, Vol. 15, pp. 606-617, 2008.

[14] T J Lewis "A Model for Nano-composite Dielectrics under Electrical stress" Intern. Conf. on Solid Diel., Winchester, UK, July 8-13, pp. 11-14, 2007.

[15] J. K. Nelson and Y. Hu "Candidate Mechanisms Responsible for Property Changes in Dielectric Nanocomposites" IEEE Conf. on Prop. \& Appl. of Diel. Mat., pp. 150-154, 2006.

[16] R. Sarathi, R.K. Sahu and T. Tanaka "Understanding the Hydrophobic Characteristics of Epoxy Nanocomposites Using Wavelets and Fractal Technique" IEEE Trans. on Diel. and Elec. Ins., Xian, China Vol. 15, pp.178186, 2008.

[17] M. F. Frechette, M. L. Trudeau, H. D. Alamdari and S. Boily "Introductory Remarks on Nanodielectrics" IEEE Tran. on Diel. and Elec. Ins. Vol. 11, pp.808-818, 2005.

[18] T. J Lewis "Nanometric Dielectrics" IEEE Tran. on Diel. and Elec. Ins. Vol. 1, pp. 812-825, 1994.

[19] T. Tanaka "Interpretation of Several Key Phenomena Peculiar to Nano Dielectrics in terms of a Multi-core Model" CEIDP, Kansas City, Mo, USA, pp. 298-301, 2006

[20] T. Maeno, T. Futami, H. Kushibe, T. Takada and C. M. Cooke, "Measurement of spatial charge distribution in thick dielectrics using the pulsed electroacoustic method" IEEE Tran. on Elec. Ins. Vol. 23, pp. 433 439, 1988.

[21] G. Chen "Interfaces and Space Charge in Polymeric Insulating Materials" (invited). In the proceedings of: MRS Fall, Symposium, Proceedings 889, Warrendale, PA, 2006.

[22] G. Chen, C. Zhang and G. Stevens, "Space charge in LLDPE loaded with nanoparticles" CEIDP, Vancouver, Canada, pp. 275 - 278, 2007. 